\title{
La variabilité des références dans les dénominations de couleur françaises
}

\author{
Grimaldi Claudio ${ }^{1, *}$ \\ ${ }^{1}$ Università degli Studi di Napoli "Parthenope", Dipartimento di Studi Economici e Giuridici, via \\ Generale Parisi 13, 80132 Naples, Italie
}

Résumé. Le concept de couleur a été depuis toujours au carrefour de plusieurs champs de connaissance tels que, entre autres, la physique, la psychologie, l'anthropologie, la physiologie et la linguistique. En particulier, le concept de couleur a été analysé de manière significative en linguistique (morphologie, sémantique, pragmatique), linguistique cognitive, psycholinguistique, traduction et lexicographie sous plusieurs angles et perspectives. Toutefois peu d'études ont analysé les évolutions en diachronie des dénominations de couleur, notamment en ce qui concerne la sphère sémantique relative aux références utilisées dans les dénominations de couleur mêmes. À partir du constant que les dénominations de couleur sont impliquées dans des phénomènes de néologisation qui enrichissent le lexique chromatique, notre contribution propose une réflexion sur quelques questions de sémantique lexicale concernant le figement des dénominations de couleur, ainsi que la variabilité en diachronie des références utilisées en français dans ces mêmes dénominations. Notre échantillon a été créé à partir de pratiques disparates de l'univers coloré (mode vestimentaire, nuanciers web, nuancier RAL, nuanciers Caran d'Ache et Faber Castell). D'un point de vue théorique, notre analyse s'insère dans le sillage des études de Dubois (Dubois Grinevald 1999; Dubois, Colette 2003 ; Dubois, Cance 2012), qui insistent sur la variabilité des dénominations de couleurs à travers des enquêtes faites grâce à des dispositifs techniques différents, ce qui reflète la diversité des processus de conceptualisation des couleurs mêmes.

\begin{abstract}
The variability of references in French color denominations. The concept of color has always interested several researchers from different fields such as, among others, physics, psychology, anthropology, physiology and linguistics. In particular, linguistics (morphology, semantics, and pragmatics), cognitive linguistics, psycholinguistics, translation and lexicography have analyzed the concept of color from several angles and perspectives. However, few studies have explored the diachronic evolutions of color denominations, especially with respect to the semantic sphere relative to the references used in the denominations of color. Starting from the consideration that the color denominations are involved in neologization phenomena, our research aims to propose a semantic reflection on the diachronic variability and figement of the references used in French color names from a sample of
\end{abstract}

\footnotetext{
*Corresponding author : claudio.grimaldi@uniparthenope.it
} 
different denominations. We have created this sample from disparate practices of the chromatic universe (fashion, web color charts, RAL color charts, Caran d'Ache and Faber Castell color charts). From a theoretical point of view, our study shares the reflections proposed in Dubois' works, which emphasize the variability of color names through surveys made using different technical devices, which takes into account the diversity of the processes involved into the conceptualization of the colors.

\section{Introduction}

Les recherches menées sur les couleurs au $\mathrm{XX}^{\mathrm{e}}$ siècle ont abordé, entre autres, les aspects principaux concernant, d'une part, la catégorisation des concepts de couleur aux niveaux cognitif et anthropologique et, de l'autre, l'arrangement linguistiques des composantes des dénominations du lexique chromatique.

En psycholinguistique et anthropologie, les travaux de Berlin et Kay (1969) constituent le point de départ de la plupart des réflexions scientifiques de la seconde moitié du $\mathrm{XX}^{\mathrm{e}}$ siècle quant à la conception de l'existence d'une liste de noms de couleurs élémentaires, appelés en anglais basic color terms, en nombre de onze, communs à toutes les langues, et de dénominations linguistiques qui sont le meilleur exemple d'une nuance de couleur, indiquées comme focal color terms. Berlin et Kay ont également interrogé la présence d'une hiérarchie conceptuelle de couleurs qui se reflète dans une construction linguistique des termes de couleur (désormais TC) en sept stades différents.

Ces réflexions ont été partiellement remises en cause surtout en ce qui concerne l'universalité des dénominations de couleur et le statut des couleurs en tant que concepts universaux. Dans sa théorie des prototypes, Rosch Heider $(1972,1973)$ reconnaît aux couleurs focales le statut de prototypes puisqu'elles sont considérées comme étant plus expressives et marquées par rapport à d'autres expressions lexicales chromatiques. En revanche, Wierzbicka $(1996,2005)$ n'introduit pas le concept de couleur dans sa liste des primitifs sémantiques puisqu'il existe des langues dans lesquelles les TC sont absents. En raison de cela, Wierzbicka formule une nouvelle théorie du prototype, où ce concept appliqué à la couleur est à considérer indique un objet ou un phénomène naturel qui exemplifie mieux la couleur même.

En linguistique cognitive contemporaine, Dubois (2006a, 2006b) souligne les limites des recherches menées par Berlin et Kay. Selon Dubois, les supports techniques et les échantillons chromatiques analysés par les deux chercheurs américains ne permettaient pas d'analyser la diversité des processus de conceptualisation de la couleur, ainsi que la pluralité des dénominations linguistiques. Les études de Dubois ont montré comment le paradigme élaboré par Berlin et Kay opérait dans un domaine de validité restreint, à savoir le cadre de référence scientifique occidental. À partir de dispositifs techniques très divers tels que, entre autres, les nuanciers des peintures pour artistes et des peintures décoratives, les teintures de vernis à ongles et cheveux, Dubois propose un modèle d'analyse plus large des dénominations de couleur élaboré à partir de pratiques techniques différentes. Ses enquêtes prennent en considération comment les locuteurs encadrent psychologiquement la catégorisation des couleurs selon des pratiques diverses et ils créent des dénominations adaptées à ces pratiques. En d'autres termes, quant au phénomène de la couleur, il faut inscrire l'étude de sa dénomination dans la diversité des pratiques qui lui donnent sens. Dans cette filière d'étude, la prise en considération de la diversité des champs d'application de la couleur permet de cerner les relations entre catégories cognitives et catégories lexicales, ainsi que la variété des modes de représentation et d'expression de la couleur d'un point de vue linguistique (Molinier 2001, 2006 ; Golka 2014 ; Krysolova 2005 ; Rodríguez Pedreira 2002). Dans plusieurs de ses travaux, Dubois fait référence à la 
dimension culturelle implicite dans la catégorisation cognitive des couleurs et dans la création des dénominations linguistiques du lexique chromatique.

À ce propos Mollard-Desfour (2008, 2011, 2017 ; Mollard-Desfour, Krylosova, Schindler 2010) et d'autres chercheurs ont analysé la symbolique dans plusieurs typologies textuelles, y compris celles littéraires (Ripoll 2018, 2019), et les origines historiques et étymologiques des mots, locutions et expressions de couleur en langue française. En ce qui concerne les analyses de Mollard-Desfour (1998, 2000, 2002, 2005, 2008, 2012, 2015), ses volumes sur le vert, le bleu, le noir, le blanc, le rouge et le gris, ainsi que les travaux de Pastoureau (2002, 2008, 2013, 2016), sont désormais des études de lexicographie incontournables sur l'histoire et la lexicalisation des dénominations et expressions de la couleur en français.

Finalement, étroitement liés à cette dernière filière d'études sur les dimensions culturelle et historique des noms de couleur, d'autres travaux menés notamment dans le domaine de la traductologie (Kristol Andres 1978 ; Silvestre, Cardeira, Villalva 2006 ; Mollard-Desfour 2008) ont mis en relief les difficultés traductives des dénominations de couleur en raison des implications culturelles et symboliques qui sont souvent à la base de la naissance des expressions de la couleur dans une langue.

Les recherches citées constituent, à notre avis, un point de départ indispensable pour toute réflexion sur les dénominations linguistiques de couleur, ainsi que sur la catégorisation et la conceptualisation des couleurs dans les différentes langues. Ces prémisses théoriques relatives aux études principales menées sur le concept de couleur nous conduisent à nous interroger sur quelques questions de sémantique lexicale concernant le figement des dénominations de couleur, ainsi que la variabilité en diachronie des références utilisées en français dans ces mêmes dénominations. En particulier, le premier objectif de nos recherches est d'analyser le degré de figement des éléments linguistiques composant des dénominations de couleur ayant une structure lexicale complexe (à deux ou trois lexèmes ou à plusieurs items comportant au moins un TC). D'un point de vue théorique, nos recherches s'insèrent dans le sillage des études de Dubois (Dubois, Grinevald 1999 ; Dubois, Colette 2003), qui insistent sur la variabilité des dénominations de couleur et sur l'inscription matérielle des couleurs dans les dispositifs de présentation de la couleur afin de prendre en considération les différents processus de sémiotisation qui construisent les couleurs comme connaissances (Dubois, Cance 2012). Un second objectif est aussi d'explorer le caractère néologique de certaines dénominations de couleur et leur éventuelle variabilité en diachronie à l'aide de quelques dictionnaires de référence sur les couleurs (Adams 2017 ; Guillemard 1998). Nos analyses ont été menées à partir d'un corpus linguistique constitué de syntagmes tirés de différentes pratiques du monde chromatique qui obéissent en partie à des objectifs commerciaux (mode vestimentaire, nuanciers web, nuancier RAL, nuanciers Caran d'Ache et Faber Castell), mais qui peuvent offrir des cas d'étude intéressants pour proposer des réflexions sémantiques valables pour toute dénomination de couleur.

\section{La construction d'un modèle d'analyse des dénominations de couleur}

Les travaux que nous avons menés dans les dernières années (Grimaldi 2017 ; Grimaldi sous presse) ont analysé les dénominations de couleur en français contemporain par rapport aux modalités linguistiques d'arrangement des composantes des structures polylexicales analysées et des référents employés dans les dénominations. Ces dénominations s'avèrent être des « étiquettes verbales » associées à chaque couleur dans les nuanciers et constituent un corpus linguistique attesté, pour le dire avec Dubois et Cance (2012 : 78), un ensemble de «élément[s] d'un corpus d'un genre particulier, une liste de formes assurant la dénomination $[\ldots]$ dans un discours que l'on peut qualifier de paradigmatique ». 
Dans une première étude, nous avons exploré une centaine de dénominations de la pratique de la mode vestimentaire repérées dans un corpus d'articles de revues de mode féminine en ligne. Notre étude a mis en valeur la présence de constructions dénominatives polylexicales qui d'un point de vue formel sont les plus fréquentes dans la pratique de la mode vestimentaire. À partir de ces premiers résultats, nous avons vérifié les structures dénominatives françaises les plus représentatives du lexique chromatique à l'aide de certaines ressources lexicographiques de référence (Adams 2017 ; Guillemard 1998). Notre but était de construire un modèle d'analyse qui comprend des structures simples à deux ou trois lexèmes et des structures d'extension à plusieurs items comportant un TC. Le tableau suivant propose une schématisation des constructions polylexicales repérées.

Tableau 1. Inventaire des dénominations polylexicales de couleur à deux et trois lexèmes.

\begin{tabular}{|l|l|}
\hline \multicolumn{1}{|c|}{ Constituantes des dénominations de couleur } & \multicolumn{1}{|c|}{ Exemples } \\
\hline $\mathrm{TC}+$ adjectif & $\begin{array}{l}\text { bleu vif, fuchsia éclatant, gris clair, jaune anisé, } \\
\text { rouge incandescent, rouge sensuel, violet } \\
\text { flamboyant, violet osé }\end{array}$ \\
\hline $\mathrm{TC}+$ nom commun & $\begin{array}{l}\text { bleu marine, brun seigle, jaune canari, jaune } \\
\text { poussin, rose barbie, vert pomme }\end{array}$ \\
\hline $\mathrm{TC}+$ plusieurs adjectifs & $\begin{array}{l}\text { gris clair subtil et lumineux, voire irisé ou } \\
\text { scintillant, beige trendy et délicat }\end{array}$ \\
\hline $\mathrm{TC}+\mathrm{TC}$ & rose fuchsia, bleu gris, vert bronze \\
\hline $\mathrm{TC}+$ nom commun + adjectif & jaune bouton d'or lumineux \\
\hline $\mathrm{TC}+$ préposition + nom commun & vert d'eau \\
\hline $\mathrm{TC}+\mathrm{TC}+$ adjectif & bleu turquoise pastel, bleu turquoise flashy \\
\hline $\mathrm{TC}+$ nom propre & bleu klein \\
\hline Nom commun $+\mathrm{TC}$ & ocre jaune \\
\hline
\end{tabular}

Ce modèle regroupe les typologies de construction des dénominations de couleur typiques de la langue française et il peut être exploité dans toute sorte d'analyse des dénominations de couleur des diverses pratiques de production d'objets colorés. Notre première recherche a permis de construire ce modèle d'analyse et de recenser la présence de dénominations néologiques dans la pratique de la mode vestimentaire féminine (emprunts à la langue anglaise, recours à l'adjectivation, choix de nouveaux référents non lexicalisés).

Notre deuxième recherche de nature sémantique a été menée sur l'extension des dénominations de couleur contenues dans un échantillon de syntagmes créé à partir de l'analyse de deux sites Internet concernant la pratique des nuanciers de couleur web. Notre hypothèse de départ portait sur la présence d'une sorte de variabilité et d'instabilité dans les dénominations de couleur à deux lexèmes dans lesquelles l'agencement des composantes $\mathrm{TC}+$ adjectif/nom commun peut être analysé sur le plan sémantique. Il se peut, en effet, que dans les dénominations polylexicales à deux lexèmes on fasse référence soit à de nouveaux référents de la réalité soit à des référents déjà utilisés dans d'autres dénominations, à savoir des dénominations où le figement entre les éléments linguistiques qui les composent n'est que partiel. Notre recherche a mis en relief des phénomènes autant d'extension des dénominations du point de vue sémantique que d'extension du point de vue lexical, ce qui nous a permis d'explorer la syntaxe particulière des syntagmes impliquant un TC en langue française.

Dans cette seconde enquête notre échantillonnage à partir des sites web étudiés a permis d'isoler environ 500 dénominations de couleur dont la présence dans les dictionnaires consultés (Adams 2017 ; Guillemard 1998) a été ensuite validée. Cette opération a permis de vérifier le degré de lexicalisation de ces dénominations en français, tout en ne prenant pas en considération les phénomènes néologiques concernant les dénominations 
polylexicales de couleur faisant l'objet d'une omission du TC (entre autres, jaune citron $>$ citron ou noir fumée $>$ noir). En particulier, notre but a été d'analyser les structures du type $\mathrm{TC}+$ adjectif et $\mathrm{TC}+$ nom commun afin de vérifier la présence de dénominations nouvelles - en raison du figement faible existant entre le TC et l'adjectif/le nom commun - étant le résultat du recours à de nouveaux référents inexploités dans le champ coloré, ainsi que l'extension de références déjà associées à des noms de couleur, appliquées à d'autres noms de couleurs.

Notre échantillon de dénominations polylexicales néologiques comptait une vingtaine de dénominations présentant les structures $\mathrm{TC}+$ nom commun et $\mathrm{TC}+$ adjectif absentes dans les ressources lexicographiques consultées. Quant aux dénominations du type TC + adjectif, celles-ci étaient les moins nombreuses et en nombre de huit : bleu égyptien, bleu givré, jaune impérial, vert impérial, blanc lunaire, jaune auréolin, vert militaire et bleu maya.

Les autres dénominations regroupées étaient du type $\mathrm{TC}+$ nom commun, dans lesquelles le nom commun est souvent un élément issu du monde botanique et zoologique. Les dénominations dont la structure des composantes est du type $\mathrm{TC}+$ nom commun étaient les suivantes : vert lichen, vert printemps, vert sauge, vert empire, bleu charrette, bleu charron, bleu guède, bleu sarcelle, noir charbon, rouge grenadine, vert lime, vert opaline et vert mélèze.

Dans notre échantillon de dénominations du type $\mathrm{TC}+$ adjectif et $\mathrm{TC}+$ nom commun, nous avons isolé une dizaine de dénominations dans lesquelles les adjectifs et les noms communs exploités sont déjà présents dans d'autres expressions lexicalisées du champ coloré. Pour ces expressions, le référent n'est pas nouveau et il s'agit plutôt d'une association nouvelle entre TC et référents utilisés dans d'autres structures polylexicales. Des exemples repérés de ce procédé sont les dénominations suivantes : jaune indien (dénominations lexicalisées : rose/rouge indien), bleu paon (dénomination lexicalisée : vert paon), jaune/vert (de) cobalt (dénominations lexicalisées : bleu/violet (de) cobalt), rouge anglais (dénomination lexicalisée : vert anglais), rouge alizarine (dénomination lexicalisée : jaune (d')alizarine), bleu dragée (dénomination lexicalisée : rose dragée), jaune chartreuse (dénomination lexicalisée : vert chartreuse), jaune olive (dénomination lexicalisée : vert olive), bleu fumée (dénominations lexicalisées : gris/noir (de) fumée).

Grâce à cette analyse, nous avons vérifié notre hypothèse de départ, à savoir le caractère instable des dénominations de couleur, qui, n'étant pas figées dans la langue, peuvent évoluer, disparaître et contribuer à la naissance de nouvelles dénominations par le biais du recours à des référents et des éléments grammaticaux déjà lexicalisés. Dans la totalité des cas analysés, nous avons remarqué que ces phénomènes lexicaux concernent des dénominations ayant comme tête du syntagme les basic color terms de Berlin et Kay. Ce constat permet d'affirmer que les TC de base se prêtent mieux à la possibilité d'être associés à de nouveaux référents ou à des adjectifs déjà lexicalisés dans le lexique chromatique, en raison du fait que leur nuance doit être spécifiée et les caractéristiques chromatiques des référents peuvent apporter des informations significatives par rapport à la nuance à désigner.

\section{La variabilité des dénominations de couleur dans les pratiques de l'univers chromatique}

Dans cette contribution nous proposons d'autres données résultant d'un travail sur deux autres pratiques du monde coloré afin d'explorer la présence en diachronie de certaines dénominations de couleur et l'emploi des référents dans ces mêmes dénominations. Nous avons éliminé de notre analyse les dénominations de couleur monolexicales, à savoir celles qui se composent d'un seul TC. Le premier support étudié est le nuancier RAL, utilisé dans le choix des couleurs de peinture dans les domaines du bâtiment, de l'industrie, de la 
carrosserie et de la sécurité routière. Nous avons classifié les dénominations de couleur d'un point de vue formel et ensuite nous avons schématisé nos données, en identifiant les combinaisons de mots les plus fréquentes, le degré de lexicalisation des dénominations repérées et les divers types de néologismes rencontrés.

Le nuancier RAL compte 212 dénominations de couleur. Chaque couleur est identifiée à travers un code spécifique à quatre chiffres, dont le premier représente la teinte et les deux derniers le code de la couleur. Les teintes présentes sont celles du jaune, de l'orange, du rouge, du rose et du violet, du bleu, du vert, du gris, du brun, du blanc et du noir. Les nuances les plus nombreuses sont celles du gris et du vert, qui comptent respectivement 38 et 36 teintes, suivies de celles du jaune (30 teintes) et du rouge (25 teintes), du bleu ( 24 teintes), du brun (20 teintes) et, enfin, du blanc et du noir (14 teintes), de l'orange (13 teintes), du rose et du violet (12 teintes).

Les typologies de constructions lexicales rencontrées sont environ 10. La combinaison la plus fréquente est celle du type TC + nom commun, qui représente $47 \%$ des cas. Parmi les références les plus communes on remarque des métaux, minéraux et pierres précieuses (25 noms), tels qu'argent, or, zinc, ardoise, fer, anthracite, granit, silex, agate, quartz, des fleurs et des plantes telles que colza, gentiane, dahlia, sapin, pin, menthe et saumon, et, enfin, des aliments, comme saumon, chocolat, curry, et des fruits, comme melon, citron, olive et fraise. D'autres références sont également présentes (par exemple, jaune soleil, rouge trafic, bleu océan, gris poussière et vert bouteille).

La typologie de combinaison lexicale avec le deuxième pourcentage le plus élevé est celle de la structure $\mathrm{TC}+\mathrm{TC}$, entre autres, beige vert, orangé rouge, violet bordeaux, ou encore bleu vert et bleu violet. Ces combinaisons constituent $20 \%$ des cas des dénominations. $16,5 \%$ d'occurrences est représenté de la combinaison TC + adjectif, où les adjectifs expriment principalement l'intensité de la couleur (clair, foncé, nacré et brillant). Parmi les adjectifs les plus rares, nous citons oriental pour le rouge, ou nocturne pour le bleu.

Les autres constructions lexicales repérées sont plutôt diversifiées : il y a la typologie $\mathrm{TC}+$ préposition + nom commun, comme rouge de sécurité, employée pour chaque teinte ; $\mathrm{TC}+$ nom + adjectif, comme bleu nuit nacré ; TC + adjectif + adjectif, comme gris clair nacré. Enfin, nous avons remarqué d'autres dénominations peu communes comme vieux rose, clair brillant et télé magenta.

Parmi les dénominations rencontrées, il y en a certaines qui ne sont pas lexicalisées dans les ressources lexicographiques de référence sur les couleurs que nous avons consultées (Adams 2017 ; Guillemard 1998). Quant aux dénominations lexicalisées, elles sont en nombre de 139 nuances et représentent $66 \%$ de la totalité. La plupart d'entre elles sont les constructions qui ont recours aux noms et les constructions composées de deux TC. En revanche, en ce qui concerne les dénominations non lexicalisées, soit les dénominations de couleur sont totalement absentes dans les ressources lexicographiques consultées, soit dans une dénomination de couleur l'élément qui suit le TC se trouve dans les dictionnaires utilisés, mais il est associé à un autre TC.

Quant aux dénominations non lexicalisées, celles-ci, qui suivent principalement la structure TC + nom commun (généralement des noms de minéraux et métaux), constituent $22 \%$ du total.

Parmi ces dénominations, il y en a certaines qui méritent une réflexion plus détaillée :

- des dénominations apparaissent dans le catalogue de peintures pour artistes APYART, comme jaune narcisse, bleu pigeon, bleu distant, bleu de sécurité, violet de sécurité, vert jonc, vert mai, vert de sécurité, gris de sécurité, brun de sécurité, blanc papyrus, mais elles ne sont pas présentes dans les dictionnaires consultés ;

- certaines teintes de gris sont utilisées par nombreuses entreprises de mobiliers en marbre et céramique, en particulier pour identifier des revêtements muraux et de sol. Ces dénominations sont, par exemple, gris béton, gris granit et gris soie; 
- la dénomination rouge oriental est utilisée par plusieurs entreprises de vernis à ongle ;

- la dénomination gentiane nacré est utilisée pour identifier un des tissus de l'entreprise de mobiliers Casamance.

Il est donc probable que les dénominations non lexicalisées dans les dictionnaires apparaissent dans d'autres pratiques du monde coloré, bien qu'elles ne soient pas si diffusées dans le lexique français. Par conséquent, cette catégorie de dénominations constitue une première typologie de néologismes puisqu'elles se basent sur des associations de constituantes complètement nouvelles. Ces types d'associations représentent les cas les plus intéressants parce que c'est la pratique en question à concevoir une nouvelle combinaison des constituantes de la dénomination même.

Les dernières données de cette analyse concernent les nouvelles dénominations issues d'un figement faible entre les éléments qui constituent ces dénominations du lexique chromatique (nouvelle association entre un TC et un référent). Ce type de résultat constitue approximativement $10 \%$ du total et dans ce cas la forme linguistique n'est nouvelle que sur le plan de l'association des éléments linguistiques qui la constituent. Parmi les dénominations les plus fréquentes qui rentrent dans cette catégorie il y a jaune olive, puisque olive est généralement associé à une teinte de vert ; orangé saumon puisque saumon est généralement lié au rose ; bleu d'eau, puisque la dénomination lexicalisée est vert d'eau, ou encore brun sépia, puisque sépia est généralement associé au noir.

Les derniers supports explorés sont les nuanciers Caran d'Ache et Faber Castell. De même que pour les études précédentes, nous n'avons analysé que les structures polylexicales non lexicalisées afin de proposer une analyse plus détaillée concernant les référents les plus employés dans les dénominations de cette pratique du monde coloré.

Un premier résultat concerne le fait que dans les nuanciers Caran d'Ache et Faber Castell la plupart des dénominations polylexicales présentent la structure $\mathrm{TC}+$ élément chimique qui est à la base de la coloration dénommée : c'est le cas, entre autres, de vert/orange de cadmium, violet de manganèse, vert oxyde de chrome, bleu/vert de phtalo(cyanine), bleu indanthrène, vert/turquoise (de) cobalt, qui ne constituent point de syntagmes intéressants sur le plan de la sémantique de la dénomination. Un seul résultat remarquable sur le plan de la variabilité en diachronie de ces dénominations concerne le pigment dénommé bleu phtalo qui, d'après Roelofs et Petillion (2012 : 136), sert pour indiquer un «bleu très lumineux [qui] se cache sous bien des appellations telles que bleu monastral, bleu hélios, bleu royal, bleu minéral, mais rarement sous le nom de bleu phtalo ». Dans une perspective diachronique, les constructions les plus intéressantes sont celles du type $\mathrm{TC}+$ nom, tels que vert réséda, brun vandyck, gris de payne, jaune paille, vert de Chine, vert de Hooker, vert pin, rouge Pompéi, ocre d'or, alizarine cramoisie, jaune de Naples, ocre de Naples, aubergine cramoisie, terre de Tolède, vert genévrier et vert de mai. Nous avons vérifié si ces dénominations se prêtent à une réflexion de nature linguistique en diachronie quant à leur utilisation dans les pratiques du monde coloré. D'après nos recherches il en ressort que certaines dénominations telles que aubergine cramoisie, ocre de Naples, terre de Tolède et vert genévrier ne semblent être réservées qu'aux dénominations des crayons Caran d'Ache et/ou Faber Castell. D'autres occurrences de ces dénominations dans d'autres nuanciers ne semblent pas être fréquemment attestées. D'autres constructions, telles que vert de Hooker, gris de payne et brun vandyck, ne sont pas très diffusées dans les nuanciers consultés, mais d'un point de vue formel leur structure se base sur un des procédés les plus productifs de la construction des dénominations de couleur, à savoir la forme TC + nom propre de l'inventeur d'une telle nuance (l'illustrateur anglais William Hooker, l'aquarelliste anglais William Payne et le peintre flamand Dick Antoon Van Dyck). En revanche, d'autres dénominations encore sont des synonymes ou des formes plus fréquentes aujourd'hui par rapport à d'autres constructions : c'est le cas de ocre d'or, qui est une appellation alternative à ocre jaune et jaune doré (Roelofs et Petillion 2012: 119), alizarine cramoisie, également nommé cramoisie de phtalocyanine ou 
rouge/rose de garance, cramoisi d'alizarine ou encore garance d'alizarine, et jaune de Naples, teinte redécouverte à l'époque de la Renaissance, initialement préparée à Naples, qui porte aussi le nom de jaune ancien. Quant à la dénomination vert pin, attestée en 1869 pour des couleurs du paysage, celle-ci s'avère être le synonyme de vert sapin, servant à désigner une teinte du champ chromatique du vert, généralement sombre, en référence à la couleur générale des arbres à feuillage persistant, notamment pins et sapins. Finalement, nous citons certaines dénominations qui, d'après des recherches effectuées sur le Trésor de la langue française informatisé (TLFi), sont lexicalisées, bien que nos ressources lexicographiques ne les recensent pas : c'est le cas de jaune paille, dénomination utilisés en tant que substantif masculin ou féminin pour indiquer la couleur elle-même, et rouge framboise. Pour cette dernière dénomination, le TLFi indique que la référence porte sur la framboise rose et non rouge, ce qui représente un autre cas intéressant de glissement de la référence originaire vers un TC qui n'est pas celui-ci qui est initialement associé au fruit.

\section{Conclusion}

Les données recueillies dans cette étude et dans les recherches déjà menées permettent de constater certaines tendances quant à la sémantique des dénominations de couleur.

Bien que partielles puisqu'elles ne concernent que certaines pratiques de l'univers chromatique (mode vestimentaire, nuanciers web, nuancier RAL, nuanciers Caran d'Ache et Faber Castell), nos analyses soulignent le caractère non figé du lexique chromatique qui ne cesse de s'enrichir à travers des procédés linguistiques toujours intéressants à analyser et à catégoriser. De ce point de vue, la catégorisation de ces procédés linguistiques et des structures polylexicales des dénominations de couleur peut contribuer à rendre compte du figement partiel de ces dénominations ainsi que des paradigmes possibles de création de nouvelles formes lexicales (simples ou composées) dans différents domaines d'application (contexte commercial, contexte scientifique ou contexte professionnel).

Quant aux résultats de nos recherches relatifs aux dénominations non lexicalisées, nous avons remarqué que pour les références nouvelles le recours à l'exploitation d'éléments botaniques ou zoologiques ayant des caractéristiques chromatiques précises représente le processus le plus productif sur le plan néologique. Toutefois, certaines références sous la forme d'adjectifs ou de noms communs requièrent une interprétation majeure de la part des locuteurs puisque leur compréhension est liée à la connaissance de certaines données historiques et culturelles encrées dans la langue française (par exemple, vert impérial, bleu charrette et vert empire). Quant au transfert d'adjectifs et noms communs déjà utilisés dans certaines dénominations du champ coloré à d'autres noms de couleur, ce phénomène se réalise en raison du fait que les référents possèdent des nuances de couleur qui ne sont pas complètement définies (par exemple, paon, olive et dragée) ou bien ils renvoient à des procédés chimiques ou possèdent des traits qui ne sont pas seulement à l'apanage de certains TC.

Quant aux nuanciers RAL, Caran d'Ache et Faber Castell, il en ressort que :

- au niveau formel, la typologie de construction lexicale la plus fréquente est celle du type $\mathrm{TC}+$ nom commun, avec une fréquence élevée de noms de minéraux, métaux et pierres précieuses, fleurs, plantes et aliments ;

- il y a deux types de dénominations néologiques. Le premier type est l'ensemble de néologismes créés à travers une association complètement nouvelle TC + nom commun (entre autres, vert jonc, blanc papyrus et jaune narcisse). Le deuxième type est l'ensemble de néologismes dans lesquels l'association est atypique, non conventionnelle, puisque des noms, des adjectifs ou des expressions particulières sont plutôt utilisés avec d'autres couleurs. Dans ce cas il s'agit de néologismes qui naissent du figement faible qui caractérise certaines dénominations chromatiques. 
Du point de vue diachronique, ce constant nous amène à prendre en considération le caractère instable et non fixe des dénominations de couleur et il nous semble que ce sont les besoins de fabrication des produits colorés à déterminer la diffusion et la lexicalisation des dénominations nouvelles, pouvant devenir communes en fonction des nécessités pratiques de dénomination. En effet, les différentes pratiques des dénominations de couleur cherchent à identifier une nuance en utilisant de divers critères, par exemple la peinture artistique utilise des désignations « techniques », qui permettent une référence univoque, tandis que la peinture décorative préfère évoquer des sensations agréables ou désagréables. Les désignations s'avèrent donc plus imprécises et floues sur le plan référentiel.

Par conséquent, sur la base des impératifs pratiques et parfois commerciaux, des dénominations de couleur peuvent facilement se lexicaliser, alors que d'autres peuvent disparaître. Si les dénominations de couleur reflètent les nécessités exprimées par les pratiques de dénomination, elles reflètent nécessairement les besoins de la société. Chaque société manifeste, au fil du temps, ses propres exigences à travers son langage et, pour le dire avec Mollard-Desfour (cité dans Gallienne 2019 : 73) « chaque société, chaque époque crée ses symboles colorés, et les mots et expressions de couleur sont autant de témoignage de la sensibilité sociale d'une époque et d'une culture, des phénomènes sociaux, de nos mentalités et notre histoire ».

\section{Références bibliographiques}

Adams, S. (2017). Le dictionnaire de la couleur. À l'usage de tous ceux qui veulent comprendre et utiliser la couleur. Paris : Pyramid éditions.

Berlin, B., Kay, P. (1969). Basic color terms: their universality and evolution. Berkeley : University of California Press.

Dubois, D., Grinevald, C. (1999). Pratiques de la couleur et dénominations. Faits de langues, 14, 1125.

Dubois, D., Colette, C. (2003). En voir de toutes les couleurs : processus de dénomination des couleurs et constructions cognitives. Vandeloise, C. (éd.). Langues et cognition. Paris : Lavoisier, 79-113.

Dubois, D., Cance, C. (2012). Vers une sémiotique du sensible : des couleurs en discours et en pratiques. Histoire Épistémologie Langage, 34-1, 63-95.

Dubois, D (2006a). Les "mots" et les catégories cognitives du sensible : des rapports problématiques. Cahiers du LCPE, 7, 23-47.

Dubois, D. (2006b). De l'expérience subjective des catégories de couleurs à l'objectivité de la couleur : approches cognitives, Cahiers du LCPE, 7, 67-78.

Eiseman, L. (2018). L'harmonie des couleurs. Édition Pantone. Paris : Pyramyd éditions.

Gallienne, A. (2019). Les 100 mots de la couleur. Paris : Presses Universitaires de France/Humensis (II édition).

Grimaldi, C. (2017). Processus de dénomination des couleurs en français contemporain : réflexion sur l'agencement des composantes dans les structures polylexicales à deux lexèmes. Annali dell'Università degli Studi di Napoli "L'Orientale"-sezione romanza, LIX-2, 127-141.

Grimaldi, C. (sous presse). Mots, couleurs et néologie : analyse des dénominations de couleur en français contemporain.

Golka, M. H. (2014). La catégorisation linguistique des couleurs : niveaux d'élémentarité des noms de couleurs français. Cognitive Studies/Études cognitives, 14, 131-147.

Guillemard, C. (1998). Le dico des mots de la couleur. Paris : Éditions du Seuil. 
Kristol, A. M. (1978). Color. Les langues romanes devant le phénomène de la couleur. Berne: Francke.

Krysolova, S. (2005). Contribution à l'étude lexico-sémantique des dénominations chromatiques en russe et en français. Thèse de doctorat sous la direction d'Antoine Nivière (Université Nancy 2), soutenue le 6 décembre 2005, à l'Université Nancy 2, Institut de russe et de serbo-croate, 789 p.

Molinier, Ch. (2001). Les adjectifs de couleur en français. Éléments pour une classification. Revue Romane, 36/2, 193-206.

Molinier Ch. (2006). Les termes de couleur en français. Essai de classification sémantico-syntaxique. Cahiers de Grammaire, 30, 259-275.

Mollard-Desfour, A. (1998, 2004). Dictionnaire des mots et expressions de couleur. Le Bleu. Paris : CNRS Editions.

Mollard-Desfour, A. (2000). Dictionnaire des mots et expressions de couleur. Le Rouge. Paris: CNRS Editions.

Mollard-Desfour, A. (2002). Dictionnaire des mots et expressions de couleur. Le Rose. Paris : CNRS Editions.

Mollard-Desfour, A. (2005). Dictionnaire des mots et expressions de couleur. Le Noir. Paris : CNRS Editions.

Mollard-Desfour, A. (2008). Dictionnaire de la couleur. Mots et expressions d'aujourd'hui. Le Blanc. Paris : CNRS Editions.

Mollard-Desfour, A. (2012). Dictionnaire de la couleur. Mots et expressions d'aujourd'hui. Le Vert. Paris : CNRS Editions.

Mollard-Desfour, A. (2015). Dictionnaire de la couleur. Mots et expressions d'aujourd'hui. Le Gris. Paris : CNRS Editions.

Mollard-Desfour, A. (2017). Les couleurs dans la mode. Phénomènes lexicaux et données sociologiques. Argod-Dutard, F. (dir.). Le français : des mots de chacun, une langue pour tous. Des français parlés à la langue des poètes. Rennes : Presses Universitaires de Rennes, 113-121.

Mollard-Desfour, A. (2008). Les mots de couleur : des passages entre langues et cultures. $<$ https://gerflint.fr/Base/Italie4/mollarddesfour.pdf $>$, dernière consultation : 27/11/2019.

Mollard-Desfour, A. (2011). Le lexique de la couleur : de la langue à la culture... et aux dictionnaires. Revue d'études françaises, 16, 89-109.

Mollard-Desfour, A., Krylosova, S., Schindler, V. M. (2010). From red bordeaux to absinthe green, from hot chocolate to cappuccino: Beverages, their referential colour terms and reflections on cultural differences. Interim Meeting "Color and Food: From the Farm to the Table " of the International Colour Association, Actes du Colloque (12-15 octobre 2010, Mar del Plata, Argentina). Buenos Aires : Grupo Argentino del Color and Nobuko, 200-205.

Pastoureau, M. (2002). Bleu. Histoire d'une couleur. Paris : Le Seuil.

Pastoureau, M. (2008). Noir. Histoire d'une couleur. Paris : Le Seuil.

Pastoureau, M. (2013). Vert. Histoire d'une couleur. Paris : Le Seuil.

Pastoureau, M. (2016). Rouge. Histoire d'une couleur. Paris : Le Seuil.

Ripoll, E. (2018). Penser la couleur en littérature. Explorations romanesques des Lumières au réalisme. Paris : Classiques Garnier.

Ripoll, É. (2019). La couleur dans le roman des Lumières. Enjeux, emplois et évolutions. Dixhuitième siècle, 51, 77-92.

Rodríguez Pedreira, N. (2002). Recherches sur les adjectifs de couleur. Description et référenciation. L'information grammaticale, 95, 27-30. 
Roelofs, I., Petillion, F. (2012). La couleur expliquée aux artistes. Paris : Eyrolles.

Rosch Heider, E. (1972). Universals in color naming and memory. Journal of Experimental Psychology, 93(1), 10-20.

Rosch Heider, E. (1973). On the internal structure of perceptual and semantic categories. Moore, E. (éd.). Cognitive development and the acquisition of language. New York-San Francisco-London : Academic Press, 111-144.

Silvestre, J. P., Cardeira, E., Villalva, A. (2006). Colour and coulour naming: crosslinguistic approaches. Lisboa : Centro de Linguística de Universidade de Lisboa-Universidade de Aveiro.

Trésor de la langue française informatisé, <http://atilf.atilf.fr/>, dernière consultation : 27/11/2019.

Wierzbicka, A. (1996). Semantics: primes and universals. Oxford : Oxford University Press.

Wierzbicka, A. (2005). There are no "Color Universals" but there are universals of visual semantics. Anthropological Linguistics, 47-2, 217-244. 